\title{
Evaluation of the Efficacy of Unipolar and Bipolar Spinal Dorsal Root Ganglion Radiofrequency Thermocoagulation in the Treatment of Postherpetic Neuralgia: a Retrospective Study
}

Jianjun Zhu

First Hospital of Jiaxing

Keyue Xie

First Hospital of Jiaxing

Songlei Liu

First Hospital of Jiaxing

Qiuli He

First Hospital of Jiaxing

Ge Luo

First Hospital of Jiaxing

Jiachun Tao

First Hospital of Jiaxing

Tingting Wang

Zhejiang University School of Medicine Second Affiliated Hospital

\section{Miao Xu}

First Hospital of Jiaxing

Jie Fu

First Hospital of Jiaxing

\section{Bin Huang}

First Hospital of Jiaxing

Ming Yao ( $\sim$ jxyaoming666@163.com)

First Hospital of Jiaxing

\section{Research article}

Keywords: postherpetic neuralgia, bipolar spinal root ganglion radiofrequency thermocoagulation, ovalbumin experiment in vitro

Posted Date: August 10th, 2020 
DOI: https://doi.org/10.21203/rs.3.rs-53338/v1

License: (c) (i) This work is licensed under a Creative Commons Attribution 4.0 International License. Read Full License 


\section{Abstract}

Background: At present, different views have been proposed on the radiofrequency treatment modes and parameters of radiofrequency thermocoagulation of the spinal dorsal root ganglion for the treatment of postherpetic neuralgia. It is urgent to identify a novel and more effective radiofrequency therapy for patients with postherpetic neuralgia.

Methods: A total of 60 patients who underwent radiofrequency thermocoagulation therapy for postherpetic neuralgia in the pain department of our Hospital were retrospectively reviewed from January 2013 to November 2017. According to the different surgical methods, the patients were divided into the following groups: unipolar group (CRF) and bipolar group (DCRF). Subsequently, the pain scores (NRS) were evaluated at the following specific time points: before the operation, on the $1 \mathrm{st}$ day after the operation, in 3 and 6 months after the operation and in 1 and 2 years after the operation. Moreover, the incidence of intraoperative and postoperative complications and the degree of pain relief were evaluated in order to assess the efficacy and prognosis of radiofrequency thermocoagulation in the two groups. The in vitro ovalbumin experiment was used to indicate the effects of unipolar and bipolar radiofrequency thermocoagulation.

Results: In this study, the intra-group comparison indicated that compared with the preoperative NRS, the postoperative NRS decreased significantly; the inter-group comparison demonstrated that the NRS of the DCRF group was lower than that of the CRF group at all time points from 6 months to 2 years following the operation. The total effective rate of the DCRF group was significantly higher than that of the CRF group in 2 years following the operation. The incidence of numbness in the DCRF group was higher than that noted in the CRF group at each time point following the operation. The ovalbumin experiments in vitro indicated that the effects of radiofrequency thermocoagulation were optimal when the distance between the two needles was $5 \mathrm{~mm}$.

Conclusion: Bipolar spinal root ganglion radiofrequency thermocoagulation exhibits a longer duration and higher effective rate in the treatment of postherpetic neuralgia and it's a treatment method worth promoting.

\section{Introduction}

Herpes zoster is caused by the varicella-zoster virus, which infects the dorsal root ganglion or cerebral nerve ${ }^{1,2}$. It is common in middle-aged and elderly people with impaired immunity. The main risk factors include female gender, family history and autoimmune diseases ${ }^{3}$. Although the herpes zoster vaccine has been widely used in certain countries ${ }^{2,4,5}$, recent studies have shown that the prevalence rate of this virus continues to rise and that the age of the disease exhibits a downward trend ${ }^{6,7}$. However, postherpetic neuralgia (PHN) refers to herpes zoster colonization in the dorsal root ganglion of the spinal cord. When the cellular immune function of the host is low the virus is activated again, causing inflammation and necrosis of the ganglia. Concomitantly, the activated virus can move along the peripheral nerve fibers to 
the skin to develop herpes, which is associated with pain ${ }^{1}$. Although certain patients have a certain tendency to self-heal, PHN can develop in case the virus in the body is not cleared in time and the pain in the affected area lasts for more than 3 months. Its severity usually depends on the presence of pain before the formation of the rash, the severity of the rash, the sex, age, and impaired immune function ${ }^{8-10}$.

The specific pathological process of PHN is complex and the pathogenesis is unknown. Moreover, a lack of effective treatment is present, which increases the social medical and family burden ${ }^{11}$. At present, the clinical treatment ${ }^{12}$ of PHN is mainly divided into the two following categories: drugs ${ }^{10,13,14}$ and surgery ${ }^{15,16}$. When the application of drugs is ineffective or the side effects caused are unbearable, surgery is considered ${ }^{16}$.

Radiofrequency therapy of spinal dorsal root ganglion (DRG) is considered as one of the most effective intervention methods for the treatment of PHN due to its small trauma, effective control, high safety, optimal accuracy and reliable repeatability. This method has been widely used previously ${ }^{17,18}$. Pulsed radio frequency (PRF) is considered to regulate only the nerve and therefore causes almost no nerve damage ${ }^{19}$. Radiofrequency thermocoagulation is considered to cause nerve damage, resulting in side effects, such as hypoesthesia and decreased muscle strength ${ }^{19}$. However, the effective rate of PRF alone in the clinical treatment is low and its effective time is short ${ }^{20}$.

Nowadays, PRF combined with local anesthetics and hormone injection is often used in the clinic to improve the clinical treatment effect. Overall, the pain relief rate of Radiofrequency thermocoagulation was significantly higher than that of $\mathrm{PRF}^{20,21}$. Therefore, it is urgent to seek a newer and more effective radiofrequency therapy for patients with postherpetic neuralgia. The purpose of the present study was to compare the effective rate, effective maintenance time and complications of bipolar and unipolar radiofrequency thermocoagulation of the spinal dorsal root ganglion in the treatment of postherpetic neuralgia, so as to guide clinical practice.

\section{Patients And Methods}

\section{Clinical data}

A total of 60 patients that underwent radiofrequency thermocoagulation therapy for postherpetic neuralgia in the pain department of our hospital from January 2013 to November 2017 were retrospectively reviewed. Among them, 34 males and 26 females were present, aged from 49 to 93 years, with an average age of $72.50 \pm 9.43$ years. Radiofrequency thermocoagulation of spinal dorsal root ganglion was performed by the same doctor with sufficient clinical pain management experience.

\section{Inclusion and exclusion criteria}

2.1 The following inclusion criteria were used: (1) The subjects met the clinical diagnostic criteria of postherpetic neuralgia; (2) The age of the subjects was higher than and/or equal to 18 years; (3) The diseased 
region of the herpes zoster only involved the unilateral trunk nerve, that is, the thoracic 1-12 nerve; (4) The subjects' pain numerical score (NRS) was higher than 4 when they were admitted to the hospital; (5) The course of the herpes zoster disease lasted more than 3 months; (6) During hospitalization, the subjects underwent radiofrequency thermocoagulation of the spinal dorsal root ganglion under the guidance of the CT scan (Subsidiary Table 1.); (7) All the subjects agreed and received follow-up by telephone.

2.2 The following exclusion criteria were used: (1) Patients with a history of malignant tumors; (2) Patients receiving immunosuppressive therapy; (3) Uncooperative patients with mental illness, mental retardation and confusion; (4) Patients with severe liver, kidney, heart and lung diseases; (5) Patients who had received a history of invasive treatment prior to operation; (6) Patients who withdrew from the operation due to lack of cooperation with the surgeon during the operation; (7) Patients who received other invasive treatment or other surgeries following the operation; (8) Patients who lacked basic information and could not be followed up following the operation.

The patients were selected according to the inclusion and exclusion criteria and all patients were divided into the bipolar group (DCRF group, $n=24$ ) and the unipolar group (CRF group, $n=36$ ) by reviewing their intraoperative imaging data.

\section{Surgical methods}

The patients entered the CT treatment room and were laid at the prone position on the treatment bed. A vital sign monitor was used to connect the monitor's blood pressure, heart rate and oxygen saturation. A positioning grid was placed at the patient's preoperative mark and fixed with adhesive tape (Fig 1a. 2a). A single CT scan was performed and following the scan, the puncture route was designed using the CT software (Fig 1b. 2b-1. 2b-2). After using the positioning grid to determine the puncture site, local infiltration anesthesia was performed with $2 \%$ lidocaine at a volume of $0.5 \mathrm{ml}$. Under the guidance of CT, the puncture needle was used to create a puncture along the preset angle and a path to the specified position. In the CRF group, a single puncture needle was punctured to a pre-designed target (Fig 1c). In the DCRF group, two puncture needles were punctured from the different angles to the pre-designed target (Fig 2c). Following completion of the initial puncture, a CT scan was performed again to confirm the puncture site. Further adjustment of the direction of the needle was performed to the outlet of the nerve root at the ventral superior edge of the intervertebral foramen in the affected area (Fig 1d. 2d). Following successful positioning of the puncture needle, the RF device was connected and the following tests were performed: (1) the sensory electrophysiological test was carried out under $50 \mathrm{hz}$ and $0.1 \mathrm{~ms}$ and the current threshold inducing abnormal pain sensation was observed to judge the accuracy of the puncture site and record the current $(\mathrm{mA})$ at this time. (2) Subsequently, the exercise electrophysiological test was carried out under $2 \mathrm{hz}$ and $1.0 \mathrm{~ms}$ in order to observe the current threshold that could make the muscle beat in the area, which was innervated by the dorsal root ganglion and to record the current $(\mathrm{mA})$ at the same time. Subsequently, the tip position was adjusted again until the movement and sensation areas were completely covered, without affecting the normal area. Finally, the radio frequency parameters were set. The parameters of the patients in the CRF and DCRF groups were both $95^{\circ} \mathrm{C}$ and 300 seconds. 
During the operation, the reaction of the patients was observed and the incidence of the complications was assessed. A record from all the patients was made. At the end of the operation, the puncture needle was removed, sterilized and bandaged with aseptic dressing. The patients were observed for $15 \mathrm{~min}$ and subsequently returned to the ward following confirmation of their vital signs being normal.

\section{Observation and follow-up}

The preoperative data included age, sex, pain course, pain location and preoperative pain numerical rating scale ${ }^{22}$ (NRS), as well as the NRS score 1 day following the operation. These data were obtained by the inpatient information system, which is termed HTShell system 3.0. The postoperative data included NRS, pain relief, operative complications and side effects at 3 months, 6 months, 1 year and 2 years, all of which were evaluated by telephone follow-up. The medical staff in the non-operation group followed up the patients by telephone.

Pain was measured with a pain numerical rating scale, ranging from 0 (no pain) to 10 (the most unbearable pain). The degree of pain relief was evaluated according to the curative effect of the patient 2 years following the operation. The curative effect was divided into 4 grades ( $a, b, c, d)$ according to the ratio of (difference between preoperative NRS and postoperative NRS) / preoperative NRS. The following grades were defined as follows: (a): Complete Relief (CR), pain relief more than 75\%; (b): Partial relief (PR), pain relief 50 to $75 \%$; (c): Mild relief (MR), pain relief 25 to $50 \%$; (d): Unresponsive Relief (NR), pain relief less than $25 \%$. The total effective rate (\%) was defined by the following equation: $[(C R+P R+$ $M R) / n] \times 100 \%$ and the marked rate $(\%)=[(C R+P R) / n] \times 100 \%$.

In addition, the incidence of the complications, including short-term pneumothorax, such as hematoma, infection and spinal cord injury and the long-term complications, such as numbness in the corresponding innervated areas and abdominal dilatation were recorded. Moreover, the incidence of numbness and abdominal distension was calculated in the affected area.

\section{Power of the study}

The analysis of the clinical data of the patients suggested that the marked rate of 2 years after the surgery was $53.33 \%$ in the CRF group and $94.74 \%$ in the DCRF group. The power of the study was estimated to $90 \%$, with $95 \%$ confidence interval and a 2 -sided type I error of $5 \%$. Therefore, the present study required 19 patients in each treatment arm. To compensate for patients who may have been lost to follow-up, 60 patients were reviewed for the two groups. Among them, 36 patients were included in the CRF group and 24 patients in the DCRF group.

\section{Ovalbumin in vitro test}

In the in vitro ovalbumin test, the puncture needle (Subsidiary Tab 1.) and radio frequency electrode (Subsidiary Tab 1.) used in the clinic were completely immersed in the culture medium, so that the active tip of the needle was wrapped by ovalbumin (Subsidiary Fig 1a. 1b). In the CRF group, the electrode was 
placed in parallel and connected to the output socket of the radio frequency generator. However, in the DCRF group, two electrodes were placed in parallel and connected to the output socket of the radio frequency generator and the distance between the two electrodes was gradually adjusted according to the experimental requirements. When the radio frequency generator was operating, the tip temperature was kept at the target temperature of $95^{\circ} \mathrm{C}$. Following heating, the electrode was cooled to room temperature. The image of thermal coagulation of ovalbumin was obtained directly on the side and directly above the active tip of the puncture needle. A measuring ruler was placed for reference (Subsidiary video 1. 2). In the DCRF group, the distance between the two electrodes was set at 2, 3, 4, 5, 6, 7 and $8 \mathrm{~mm}$. The length, width, height and final tissue weight $(\mathrm{mg})$ of the thermal coagulation in the CRF and DCRF groups were recorded by manual measurement of thermal coagulation at different intervals and different time points (Fig 3a. 3b. 3c. 3d). Therefore, the effects of bipolar distance and heating time on the size of coagulation were analyzed further.

\section{Statistical analysis}

The SPSS25.0 (Subsidiary Tab 1.) was used for statistical analysis. The frequency was used as the main variable for the classification of the groups. The normality of the distribution was tested by the Kolmogorov-Smirnov test. The variables of the non-normal distribution were presented by the median (Quartile spacing) and were compared by the Mann-Whitney $U$ test. The variables of the normal distribution were analyzed and compared by the independent sample $T$ test and the values were expressed as mean \pm standard deviation ( $\mathrm{X} \pm \mathrm{SD}$ ). The analysis was performed using the Pearson's chisquared or the Fisher exact tests as appropriate. Bilateral $\mathrm{P}<0.05$ indicated that the differences was statistically significant.

\section{Results}

\section{Clinical data of patients}

A total of 60 patients participated in the present study, of which 11 patients were lost to follow-up following operation. A total of 6 and 5 patients were lost to follow-up in the CRF and DCRF groups, respectively. The total loss of the follow-up rate was $18.33 \%$. The comparison of the basic demographic and clinical data of the two groups prior to the operation indicated no significant differences between the two groups in terms of sex, age, course of pain, location of pain and NRS prior to the operation (Tab 1).

\section{Assessment of the success of surgical puncture}

\subsection{Electrophysiological test of the CRF and DCRF groups}

The results of the electrophysiological test indicated that the sensory test of the CRF group was 0.39 $\pm 0.12(\mathrm{~mA})$, whereas the sensory test of the DCRF group was $0.36 \pm 0.11(\mathrm{~mA}), P=0.39$; The exercise test of the CRF group was $0.41 \pm 0.14(\mathrm{~mA})$ and the exercise test of the DCRF group was $0.34 \pm 0.11(\mathrm{~mA})$, $P=0.07$. The difference was not significant (Subsidiary Tab 2). 


\section{$2.23 \mathrm{D}$ reconstruction image}

Following CT scanning, the path of the puncture needle in the dorsal root ganglion was visually verified by the three-dimensional reconstruction technique and the image of the successful puncture was shown (Fig 1e. 2e).

\section{Pain score (NRS)}

3.1 Comparative analysis of NRS prior to and following operation in the CRF group

The preoperative NRS and postoperative 1 day, 3 month, 6 month, 1 year and 2 year NRS in the CRF group are shown in Table 2. The postoperative NRS score at each time point was lower than that prior to the operation. The difference was significant (Tab 2).

3.2 Comparative analysis of NRS prior to and following operation in the DCRF group

The preoperative NRS and postoperative 1 day, 3 month, 6 month, 1 year and 2 year NRS in the DCRF group are shown in Table 2. The postoperative NRS score at each time point was lower than that prior to the operation and the difference was statistically significant (Tab 2).

3.3 Comparative analysis of NRS prior to and following operation between the CRF and DCRF groups

No significant differences were noted in the NRS score between the DCRF and CRF groups prior to the operation and on the 1st day and 3 months following the operation. However, at 6 months following the operation, the NRS score of the DCRF group was significantly lower than that of the CRF group. This effect was noted for the first time. Subsequently, at all the time points from 6 months to 2 years following the operation, the NRS score of the DCRF group was significantly lower than that of the CRF group (Fig 4).

\section{Pain relief degree}

From the postoperative follow-up, at 2 years following the operation, the marked rate of the CRF group $(53.33 \%)$ was significantly lower than that of the DCRF group $(94.74 \%)$. No significant differences were noted in the marked rate at other time points. The total effective rate of the CRF and DCRF groups at each time point following operation is shown in Table 3. No significant differences were noted between the two groups (Tab 3).

\section{Operative complications}

The patients in both groups completed the operation successfully during hospitalization. The main operative complications were pneumothorax, spinal cord injury, hematoma, infection, numbness and abdominal distension. The review of the disease history of the patients demonstrated no intraoperative complications in the aforementioned two groups. 
No short-term postoperative complications were noted in both groups. However, observation of long-term postoperative complications demonstrated that all patients exhibited varying degrees of skin numbness in the corresponding innervation area, whereas certain patients had abdominal distension. No other serious or permanent complications and side effects were observed during the follow-up period. The statistical results of the incidence of long-term postoperative complications are shown in Table 4. Significant differences were noted in the incidence of numbness at each time point between the two groups, whereas no significant difference was noted in the incidence of abdominal distension between the two groups (Tab 4).

\section{Ovalbumin in vitro test}

6.1 The length, width and height of coagulation at different time points of the ovalbumin in vitro test in each group were measured as shown in Figs $3 a$. $3 b$ and $3 c$. The results of the length measurement indicated no difference in the final length of each group, while the width and height of each group indicated that the width and height of the $5 \mathrm{~mm}$ group was the widest and highest (Fig 3a. 3b. 3c).

6.2 The final coagulant weight of each group of the ovalbumin in vitro test is shown in figure $3 \mathrm{~d}$. According to the figure, when the distance between the two electrodes was $5 \mathrm{~mm}$, the thermal solidification effect was the best and the weight of the coagulum was the highest, reaching 818.57 $\pm 26.02 \mathrm{mg}$ (Fig 3d).

Based on the above experimental results of the ovalbumin in vitro test, it is inferred that when the distance between the two electrodes was $5 \mathrm{~mm}$, the effects of radiofrequency thermocoagulation was optimal. This finding has instructive significance for radiofrequency thermocoagulation of bipolar spinal dorsal root ganglion in the treatment of postherpetic neuralgia.

\section{Discussion}

In recent years, radiofrequency therapy has been widely recognized due to its high success rate, optimal efficacy, high safety and high patient satisfaction. It has gradually become one of the mainstream methods for the treatment of neuralgia. Previous research studies on its mechanism have been carried out gradually.

The mechanism of PRF has been investigated by previous studies that demonstrated by electron microscopy the ultrastructural damage of axons, abnormal production of membranes and mitochondria and the collapse of microtubules and microfilaments following $\mathrm{PRF}^{23}$. Previous studies have shown that PRF can regulate the synaptic plasticity of nerve cells through in vivo experiments and have speculated that the analgesic effect of PRF may be achieved through neuro-regulation ${ }^{19}$. CRF produces highfrequency current, induces ion oscillation in tissues, heats up tissues, increases local temperature and inactivates hypersensitive nerve endings caused by pathological changes through thermal effect, so as to achieve the purpose of the treatment. At present, it is believed that the analgesic effect of PRF may be due to the temporary blocking of neural signals by neurons through the nerve conduction pathway, while 
the analgesic effect of CRF may be through the permanent blocking of neural signals through the neural pathway ${ }^{19}$.

At present, in the clinic, the radio frequency is used to treat neuralgia ${ }^{20}$ and has been used extensively, such as in the case of paroxysmal collective headache ${ }^{24}$, primary trigeminal neuralgia ${ }^{21,25-27}$, glossopharyngeal neuralgia ${ }^{28}$, postherpetic neuralgia ${ }^{15,17,18,29}$, chronic knee pain ${ }^{16}$ and postherpetic trigeminal neuralgia ${ }^{30}$. In addition, certain studies on the use of different radio frequency modes for the treatment of neuralgia have been carried out consecutively. Previous studies have shown that the effect of simple pulsed radiofrequency is not successful, whereas the therapeutic effect of pulsed radiofrequency combined with radiofrequency thermocoagulation is suitable for its clinical use $\mathrm{e}^{26,27}$. In the comparative study of unipolar and bipolar groups, it was found that the bipolar group exhibited more advantages in pain relief and functional recovery ${ }^{16,21}$. In the clinical treatment of postherpetic neuralgia, the discussion and research of unipolar and bipolar radiofrequency have not been reported. Firstly, in the present study, the electrophysiological test was used to evaluate whether the puncture was in place for clinical applications. The sensory test parameters were $50 \mathrm{hz}$ and $0.1 \mathrm{~ms}$ and the exercise test parameters were $2 \mathrm{hz}$ and $1.0 \mathrm{~ms}$. The data demonstrated that when the electrophysiological test results were within $1.0 \mathrm{~mA}$, the puncture needle was close to the target ganglion, suggesting that the puncture was in place. The overall puncture process is roughly as shown in Figs. 1 to 3. Initially routine puncture preparation was performed and subsequently the pre-designed puncture route was used. Finally, the puncture was performed according to the designed route to reach the target site.

Secondly, the data indicated that several patients were treated with unipolar radiofrequency thermocoagulation. By using the postoperative follow-up of the patients who underwent radiofrequency thermocoagulation of the dorsal root ganglion, the data indicated that the NRS of the patients in the unipolar and bipolar groups was significantly lower than that noted prior to the operation. We believe that this was due to the relief of the postoperative pain caused by local injury and due to local edema caused by early operation. Following disappearance of the edema, the degree of pain gradually stabilized. The comparison between the two groups revealed no significant differences in the NRS score between the two groups on the first day and in 3 months following the operation. However in 6 months, 1 year and 2 years following the operation, the NRS score in the DCRF group was significantly lower than that in the CRF group, indicating that the pain relief time was longer in the DCRF group. Concomitantly, the marked rate in the DCRF group was significantly higher than that in the CRF group 2 years following the operation.

In addition to the therapeutic effect, we also made a statistical analysis of the surgical complications of the patients. According to the review of the results of the CT scan during the operation, no complications were noted in both groups. For postoperative complications, the incidence of numbness in the DCRF group was higher than that in the CRF group at each time point. It is interesting to note that certain patients had abdominal distension following the operation. In addition, the occurrence of abdominal distension exhibited a distinction between the upper and lower thoracic segments, with a significant increase in the lower thoracic segments (below T8). Therefore, we conducted follow-up statistics on this 
initial result. The results indicated no significant differences in the incidence of abdominal distension between the two groups. We considered that the possible reason was the existence of ribs in the upper chest, which affected the effects of dilation; the limitation caused due to the lower thoracic segment was relatively small, therefore the bulge was more obvious and the occurrence of the abdominal distension indicated an optimal effect. However, the association between this phenomenon and the curative effects required further investigation.

However, previous studies have suggested that the extension of the time of radiofrequency exposure is not an effective method for the treatment of neuropathic pain and excessive exposure is associated with increased neuronal damage ${ }^{19,31}$. In order to assess the optimal thermal coagulation effect of bipolar more clearly, we explored the radio frequency duration and bipolar distance in the ovalbumin in vitro test 32. The in vitro ovalbumin model is often used to simulate the internal environment of the human body 21,33 . The results of the ovalbumin in vitro test indicated a difference in the effects of radiofrequency thermocoagulation between the bipolar and unipolar groups. When the distance of the two electrodes was $5 \mathrm{~mm}$, the effects of the radio frequency thermocoagulation was optimal. It is even more surprising that when the bipolar radio frequency reached approximately $120 \mathrm{~s}$, the coagulation volume of ovalbumin remained constant with time. It is uncertain whether the RF time for the patients in the clinical work can be reduced to $120 \mathrm{~s}$.

The present study exhibits certain shortcomings. First of all, it is a single-center study with relatively limited clinical samples. Secondly, in the patient follow-up, we followed-up the subjects to a maximum time duration of 2 years after the operation. Therefore, we may need to increase the follow-up time to further clarify the curative effect, such as 3 years and 5 years after the operation. Furthermore, in the ovalbumin in vitro test, we did not further design the spatial position and temperature gradient of the electrode, such as crossover, tip adjacency or tail adjacency in the three-dimensional space. The aforementioned shortcomings will be improved and optimized in the following research.

The data indicated that radiofrequency thermocoagulation of bipolar dorsal root ganglion exhibited a longer duration in the treatment of postherpetic neuralgia and that the effects of radiofrequency thermocoagulation were not optimal when the distance between the two electrodes was $5 \mathrm{~mm}$, which is helpful to guide clinical work and provide more effective treatment for patients.

\section{Conclusion}

In summary, bipolar spinal dorsal root ganglion radiofrequency thermocoagulation has a long duration and high effective rate in the treatment of postherpetic neuralgia. Therefore, it may be a new choice for the clinical treatment of postherpetic neuralgia.

\section{Abbreviations}

CRF

Page $11 / 22$ 
unipolar radiofrequency thermocoagulation therapy group; DCRF:bipolar radiofrequency thermocoagulation therapy group; NRS:Numeric rating scale; PHN:postherpetic neuralgia; DRG:spinal dorsal root ganglion; PRF:Pulsed radio frequency; CR:Complete relief; PR:Partial Relief; MR:Mild relief; NR:Unresponsive Relief; Fig:Figure; Tab:Table.

\section{Declarations}

\section{Acknowledgements}

The researchers of this project are very grateful for the understanding and support of the patients and their families who participated in this study.

\section{Authors' contributions}

Study design: Jianjun Zhu, Keyue Xie. Study investigator: Jianjun Zhu Qiuli He, Ge Luo, Jiachun Tao, Tingting Wang, Miao Xu, Jie Fu. Enrolled patients: Songlei Liu. Collection and assembly of data: Jianjun Zhu, Keyue Xie. Data analysis: Jianjun Zhu, Keyue Xie. Data interpretation: Jianjun Zhu, Keyue Xie, Bin Huang, Ming Yao. Manuscript preparation: Jianjun Zhu. Manuscript review and revisions: Jianjun Zhu, Bin Huang, Ming Yao. Final approval of manuscript: All authors. All authors reviewed and approved the final manuscript for submission.

\section{Funding}

The present study was supported by the National Natural Science Foundation of China (81901124), Natural Science Foundation of Zhejiang Province of China (LY20H090020, LGF2OH090021, LQ19H090007), Key Discipline Established by Zhejiang Province and Jiaxing City Jointly --Pain Medicine (2019-ss-ttyx) and Jiaxing Key Laboratory of Neurology and Pain Medicine.

\section{Ethics approval and consent to participate}

The present study was examined and approved by the Ethics Committee of the First Hospital of Jiaxing (LS-2010-119).

\section{Consent for publication}

Not applicable.

\section{Availability of data and materials}

All data generated or analysed during this study are included in this published article and its supplementary information files.

\section{Competing interests}

Not applicable. 


\section{References}

1. Kennedy PGE, Gershon AA. Clinical Features of Varicella-Zoster Virus Infection. Viruses. 2018;10(11):1-11.

2. Freer G, Pistello M. Varicella-zoster virus infection: natural history, clinical manifestations, immunity and current and future vaccination strategies. The new microbiologica. 2018;41(2):95-105.

3. Kawai K, Yawn BP. Risk Factors for Herpes Zoster: A Systematic Review and Meta-analysis. Mayo Clin Proc. 2017;92(12):1806-1821.

4. Cunningham AL, Levin MJ. Herpes Zoster Vaccines. J Infect Dis. 2018;218(suppl 2):S127-S133.

5. Schmader K. Herpes Zoster. Ann Intern Med. 2018;169(3):ITC19-ITC31.

6. Li JY. Herpes zoster ophthalmicus: acute keratitis. Curr Opin Ophthalmol. 2018;29(4):328-333.

7. Kawai K, Yawn BP, Wollan P, Harpaz R. Increasing Incidence of Herpes Zoster Over a 60-year Period From a Population-based Study. Clinical infectious diseases : an official publication of the Infectious Diseases Society of America. 2016;63(2):221-226.

8. Forbes HJ, Thomas SL, Smeeth L, et al. A systematic review and meta-analysis of risk factors for postherpetic neuralgia. Pain. 2016;157(1):30-54.

9. Koshy E, Mengting L, Kumar H, Jianbo W. Epidemiology, treatment and prevention of herpes zoster: A comprehensive review. Indian J Dermatol Venereol Leprol. 2018;84(3):251-262.

10. Gross GE, Eisert L, Doerr HW, et al. S2k guidelines for the diagnosis and treatment of herpes zoster and postherpetic neuralgia. Journal der Deutschen Dermatologischen Gesellschaft $=$ Journal of the German Society of Dermatology : JDDG. 2020;18(1):55-78.

11. Gudin J, Fudin J, Wang E, et al. Treatment Patterns and Medication Use in Patients with Postherpetic Neuralgia. Journal of managed care \& specialty pharmacy. 2019;25(12):1387-1396.

12. Werner RN, Nikkels AF, Marinovic B, et al. European consensus-based (S2k) Guideline on the Management of Herpes Zoster - guided by the European Dermatology Forum (EDF) in cooperation with the European Academy of Dermatology and Venereology (EADV), Part 2: Treatment. J Eur Acad Dermatol Venereol. 2017;31(1):20-29.

13. Forstenpointner J, Rice ASC, Finnerup NB, Baron R. Up-date on Clinical Management of Postherpetic Neuralgia and Mechanism-Based Treatment: New Options in Therapy. J Infect Dis. 2018;218(suppl 2):S120-S126.

14. Kaufman AR, Myers EM, Moster ML, et al. Herpes Zoster Optic Neuropathy. J Neuroophthalmol. 2018;38(2):179-189.

15. Lin CS, Lin YC, Lao HC, Chen CC. Interventional Treatments for Postherpetic Neuralgia: A Systematic Review. Pain physician. 2019;22(3):209-228.

16. Gulec E, Ozbek H, Pektas S, Isik G. Bipolar Versus Unipolar Intraarticular Pulsed Radiofrequency Thermocoagulation in Chronic Knee Pain Treatment: A Prospective Randomized Trial. Pain physician. 2017;20(3):197-206. 
17. Kim ED, Lee Yl, Park HJ. Comparison of efficacy of continuous epidural block and pulsed radiofrequency to the dorsal root ganglion for management of pain persisting beyond the acute phase of herpes zoster. PloS one. 2017;12(8):e0183559.

18. Kim K, Jo D, Kim E. Pulsed Radiofrequency to the Dorsal Root Ganglion in Acute Herpes Zoster and Postherpetic Neuralgia. Pain physician. 2017;20(3):E411-e418.

19. Cahana A, Vutskits L, Muller D. Acute differential modulation of synaptic transmission and cell survival during exposure to pulsed and continuous radiofrequency energy. The Journal of Pain. 2003;4(4):197-202.

20. Usmani H, Dureja GP, Andleeb R, Tauheed N, Asif N. Conventional Radiofrequency Thermocoagulation vs Pulsed Radiofrequency Neuromodulation of Ganglion Impar in Chronic Perineal Pain of Nononcological Origin. Pain Med. 2018;19(12):2348-2356.

21. Huang B, Xie K, Chen Y, Wu J, Yao M. Bipolar radiofrequency ablation of mandibular branch for refractory V3 trigeminal neuralgia. J Pain Res. 2019;12:1465-1474.

22. Chang AK, Bijur PE, Esses D, Barnaby DP, Baer J. Effect of a Single Dose of Oral Opioid and Nonopioid Analgesics on Acute Extremity Pain in the Emergency Department. Jama. 2017;318(17):1661-1667.

23. Erdine S, Bilir A, Cosman ER, Cosman ER, Jr. Ultrastructural changes in axons following exposure to pulsed radiofrequency fields. Pain Pract. 2009;9(6):407-417.

24. Fang L, Jingjing L, Ying S, et al. Computerized tomography-guided sphenopalatine ganglion pulsed radiofrequency treatment in 16 patients with refractory cluster headaches: Twelve- to 30-month follow-up evaluations. Cephalalgia. 2016;36(2):106-112.

25. Yao $P$, Hong $T$, Wang ZB, et al. Treatment of bilateral idiopathic trigeminal neuralgia by radiofrequency thermocoagulation at different temperatures. Medicine (Baltimore). 2016;95(29):e4274.

26. Ding Y, Li H, Hong T, et al. Combination of Pulsed Radiofrequency with Continuous Radiofrequency Thermocoagulation at Low Temperature Improves Efficacy and Safety in V2/V3 Primary Trigeminal Neuralgia. Pain physician. 2018;21(5):E545-e553.

27. Elawamy A, Abdalla EEM, Shehata GA. Effects of Pulsed Versus Conventional Versus Combined Radiofrequency for the Treatment of Trigeminal Neuralgia: A Prospective Study. Pain physician. 2017;20(6):E873-e881.

28. Song L, He L, Pei Q, et al. CT-guided percutaneous radiofrequency thermocoagulation for glossopharyngeal neuralgia: A retrospective clinical study of 117 cases. Clin Neurol Neurosurg. 2019;178:42-45.

29. Ding Y, Yao P, Li H, et al. CT-Guided Stellate Ganglion Pulsed Radiofrequency Stimulation for Facial and Upper Limb Postherpetic Neuralgia. Front Neurosci. 2019;13:170.

30. Wan C, Dong DS, Song T. High-Voltage, Long-Duration Pulsed Radiofrequency on Gasserian Ganglion Improves Acute/Subacute Zoster-Related Trigeminal Neuralgia: A Randomized, DoubleBlinded, Controlled Trial. Pain physician. 2019;22(4):361-368. 
31. Arakawa K, Kaku R, Kurita M, Matsuoka Y, Morimatsu H. Prolonged-duration pulsed radiofrequency is associated with increased neuronal damage without further antiallodynic effects in neuropathic pain model rats. J Pain Res. 2018;11:2645-2651.

32. Zhang C, Wang P, Liu A, et al. Experimental study on dose-effect relationship of bipolar radiofrequency thermocondenser in vitro. Chinese Journal of Physical Medicine and Rehabilitation. 2018;40(6):414-417.

33. LUO F, YU X-T, LI D-Y, et al. Study on the safety of frequency and temperature parameters of pulsed radio frequency by egg white experiment. Chinese Journal of Pain Medicine. 2012;18(12):742-745.

\section{Tables}

Table 1

Clinical data of patients

\begin{tabular}{|c|c|c|}
\hline & CRF & DCRF \\
\hline \multicolumn{3}{|c|}{ Age (year) } \\
\hline & $70.67 \pm 10.19$ & $72.74 \pm 8.02$ \\
\hline$P$ value & & 0.147 \\
\hline \multicolumn{3}{|l|}{ Sex } \\
\hline Male & 17 & 11 \\
\hline Female & 13 & 8 \\
\hline$P$ value & & 0.933 \\
\hline \multicolumn{3}{|c|}{ Painful area } \\
\hline & $7.71 \pm 2.53$ & $6.80 \pm 2.30$ \\
\hline$P$ value & & 0.295 \\
\hline \multicolumn{3}{|c|}{ The length of painful time (month) } \\
\hline & $10.72(2.00,6.88)$ & $8.55(2.50,12.00)$ \\
\hline$P$ value & & 0.228 \\
\hline \multicolumn{3}{|c|}{ NRS score before surgery } \\
\hline & $5.47 \pm 1.02$ & $5.27 \pm 0.69$ \\
\hline$P$ value & & 0.083 \\
\hline
\end{tabular}


Table 2

Comparative analysis of NRS prior to and following operation in the CRF group and the DCRF group

\begin{tabular}{|c|c|c|c|c|c|c|}
\hline & \multirow{2}{*}{$\begin{array}{l}\text { Before } \\
\text { Surgery }\end{array}$} & \multicolumn{5}{|c|}{ Time after surgery } \\
\hline & & 1 day & 3 month & 6 month & 1 year & 2 year \\
\hline \multicolumn{7}{|l|}{ CRF } \\
\hline & $5.27 \pm 0.69$ & $\begin{array}{l}1.83 \pm \\
0.65\end{array}$ & $\begin{array}{l}2.50 \pm \\
1.31\end{array}$ & $2.6 \pm 1.28$ & $\begin{array}{l}2.70 \pm \\
1.18\end{array}$ & $2.60 \pm 1.102$ \\
\hline $\begin{array}{l}\mathrm{P} \\
\text { value }\end{array}$ & & 0.001 & 0.001 & 0.001 & 0.001 & 0.001 \\
\hline \multicolumn{7}{|l|}{ DCRF } \\
\hline & $5.47 \pm 1.02$ & $\begin{array}{l}1.95 \pm \\
0.52\end{array}$ & $\begin{array}{l}2.21 \pm \\
0.79\end{array}$ & $\begin{array}{l}2.05 \pm \\
0.91\end{array}$ & $\begin{array}{l}2.16 \pm \\
1.02\end{array}$ & $1.95 \pm 0.85$ \\
\hline $\begin{array}{l}\mathrm{P} \\
\text { value }\end{array}$ & & 0.001 & 0.001 & 0.001 & 0.001 & 0.001 \\
\hline
\end{tabular}


Table 3

Analysis of pain relief degree

\begin{tabular}{|c|c|c|c|c|c|c|c|c|}
\hline \multirow[t]{2}{*}{ Degree } & \multicolumn{2}{|c|}{ Postoperative 3 month } & \multicolumn{2}{|c|}{$\begin{array}{l}\text { Postoperative } 6 \\
\text { month }\end{array}$} & \multicolumn{2}{|c|}{$\begin{array}{l}\text { Postoperative } \\
1 \text { year }\end{array}$} & \multicolumn{2}{|c|}{$\begin{array}{l}\text { Postoperative } \\
2 \text { year }\end{array}$} \\
\hline & CRF & DCRF & CRF & DCRF & CRF & DCRF & CRF & DCRF \\
\hline $\mathrm{CR}$ & 8 & 4 & 7 & 6 & 4 & 6 & 3 & 7 \\
\hline PR & 10 & 12 & 11 & 10 & 15 & 10 & 13 & 11 \\
\hline MR & 9 & 3 & 8 & 3 & 8 & 2 & 10 & 1 \\
\hline NR & 3 & 0 & 4 & 0 & 3 & 1 & 4 & 0 \\
\hline \multicolumn{9}{|c|}{ The marked rate (\%) } \\
\hline & 60.00 & 84.21 & 60.00 & 84.21 & 63.33 & 84.21 & 53.33 & 94.74 \\
\hline$P$ value & 0.073 & & 0.073 & & 0.115 & & 0.002 & \\
\hline \multicolumn{9}{|c|}{$\begin{array}{l}\text { The total effective rate } \\
(\%)\end{array}$} \\
\hline & 90.00 & 100 & 86.67 & 100 & 90.00 & 94.74 & 86.67 & 100 \\
\hline$P$ value & 0.273 & & 0.148 & & 1.000 & & 0.148 & \\
\hline
\end{tabular}

Table 3. Analysis of pain relief degree. The marked rate $(\%)=[(C R+P R) / n] \times 100 \%$. The total effective rate $(\%)=[(C R+P R+M R) / n] \times 100 \%$. 
Table 4

Comparisons of long-term postoperative complications between CRF and DCRF

\begin{tabular}{|c|c|c|c|c|c|c|c|c|}
\hline \multirow[t]{2}{*}{ Complications } & \multicolumn{2}{|c|}{$\begin{array}{l}\text { Postoperative } 3 \\
\text { month }\end{array}$} & \multicolumn{2}{|c|}{$\begin{array}{l}\text { Postoperative } 6 \\
\text { month }\end{array}$} & \multicolumn{2}{|c|}{$\begin{array}{l}\text { Postoperative } \\
1 \text { year }\end{array}$} & \multicolumn{2}{|c|}{$\begin{array}{l}\text { Postoperative } \\
2 \text { year }\end{array}$} \\
\hline & CRF & DCRF & CRF & DCRF & CRF & DCRF & CRF & DCRF \\
\hline \multicolumn{9}{|l|}{ Numbness } \\
\hline Yes & 19 & 17 & 18 & 17 & 16 & 16 & 8 & 13 \\
\hline No & 11 & 2 & 12 & 2 & 14 & 3 & 22 & 6 \\
\hline Incidence rate (\%) & 63.33 & 89.48 & 60.00 & 89.48 & 53.33 & 84.21 & 26.67 & 68.42 \\
\hline$P$ value & 0.043 & & 0.026 & & 0.027 & & 0.004 & \\
\hline \multicolumn{9}{|l|}{$\begin{array}{l}\text { Abdominal } \\
\text { distension }\end{array}$} \\
\hline Yes & 21 & 12 & 16 & 9 & 13 & 7 & 9 & 6 \\
\hline No & 9 & 7 & 14 & 10 & 17 & 12 & 21 & 13 \\
\hline Incidence rate (\%) & 70.00 & 63.33 & 53.33 & 47.37 & 43.33 & 36.84 & 30.00 & 31.58 \\
\hline$P$ value & 0.619 & & 0.684 & & 0.652 & & 0.907 & \\
\hline \multicolumn{9}{|c|}{$\begin{array}{l}\text { Table } 4 \text {. The incidence of long-term postoperative complications. Significant differences were noted } \\
\text { in the incidence of numbness at each time point between the two groups, } P(3 \text { month })=0.043 \text {, } \\
P(6 \text { month })=0.026, P(1 \text { year })=0.027, P(2 \text { year })=0.004 \text {. Whereas no significant difference was noted in } \\
\text { the incidence of abdominal distension between the two groups, } P(3 \text { month })=0.619, P(6 \text { month })=0.684 \text {, } \\
P(1 \text { year })=0.652, P(2 \text { year })=0.907 \text {. }\end{array}$} \\
\hline
\end{tabular}

Figures 


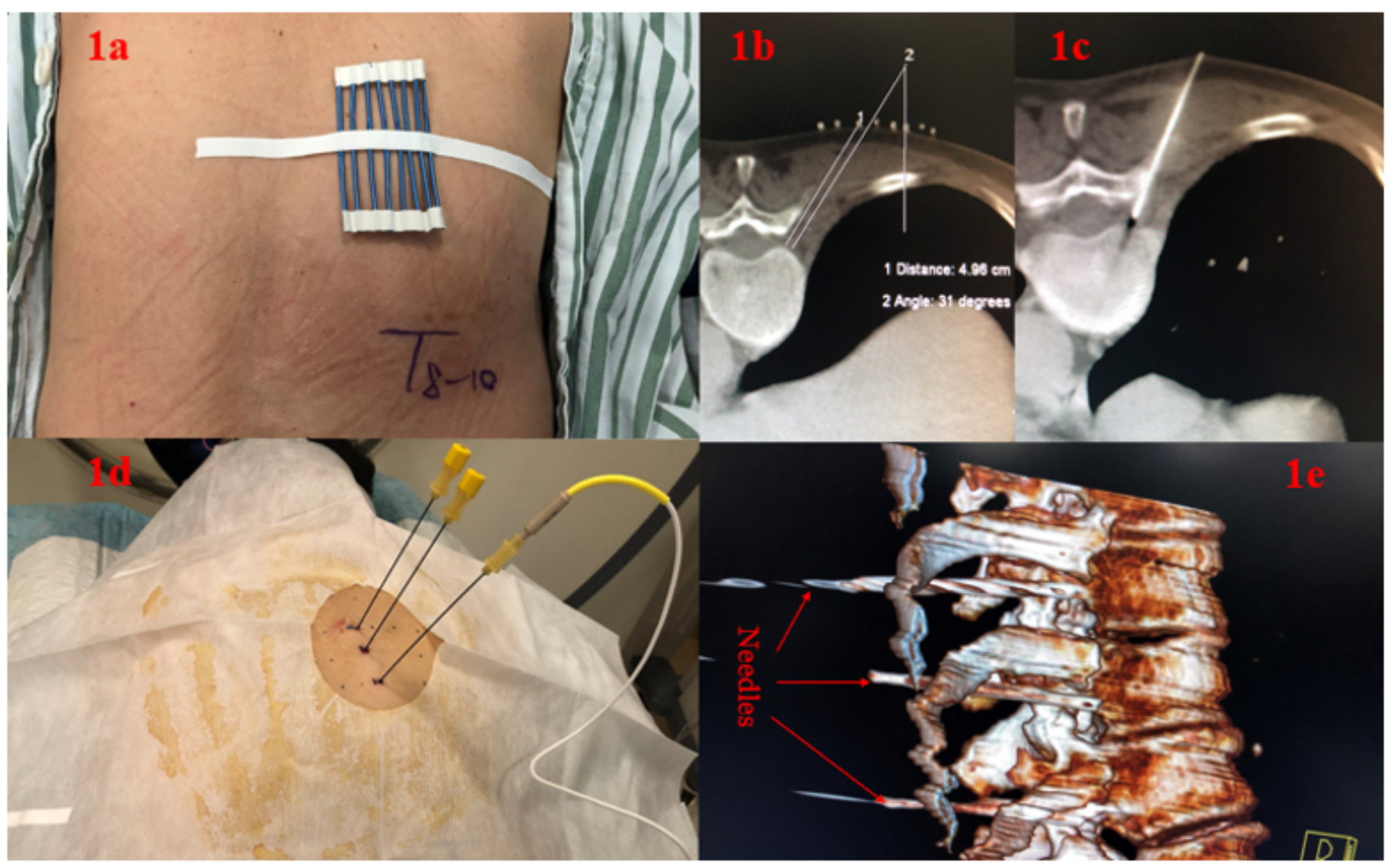

\section{Figure 1}

1a: Place positioning mark; $1 \mathrm{~b}$ : Design the puncture route; $1 \mathrm{c}$ : Puncture to predetermined position; $1 \mathrm{~d}$ : Adjust the puncture needle; 1 e:3D reconstruction image of the CRF group 


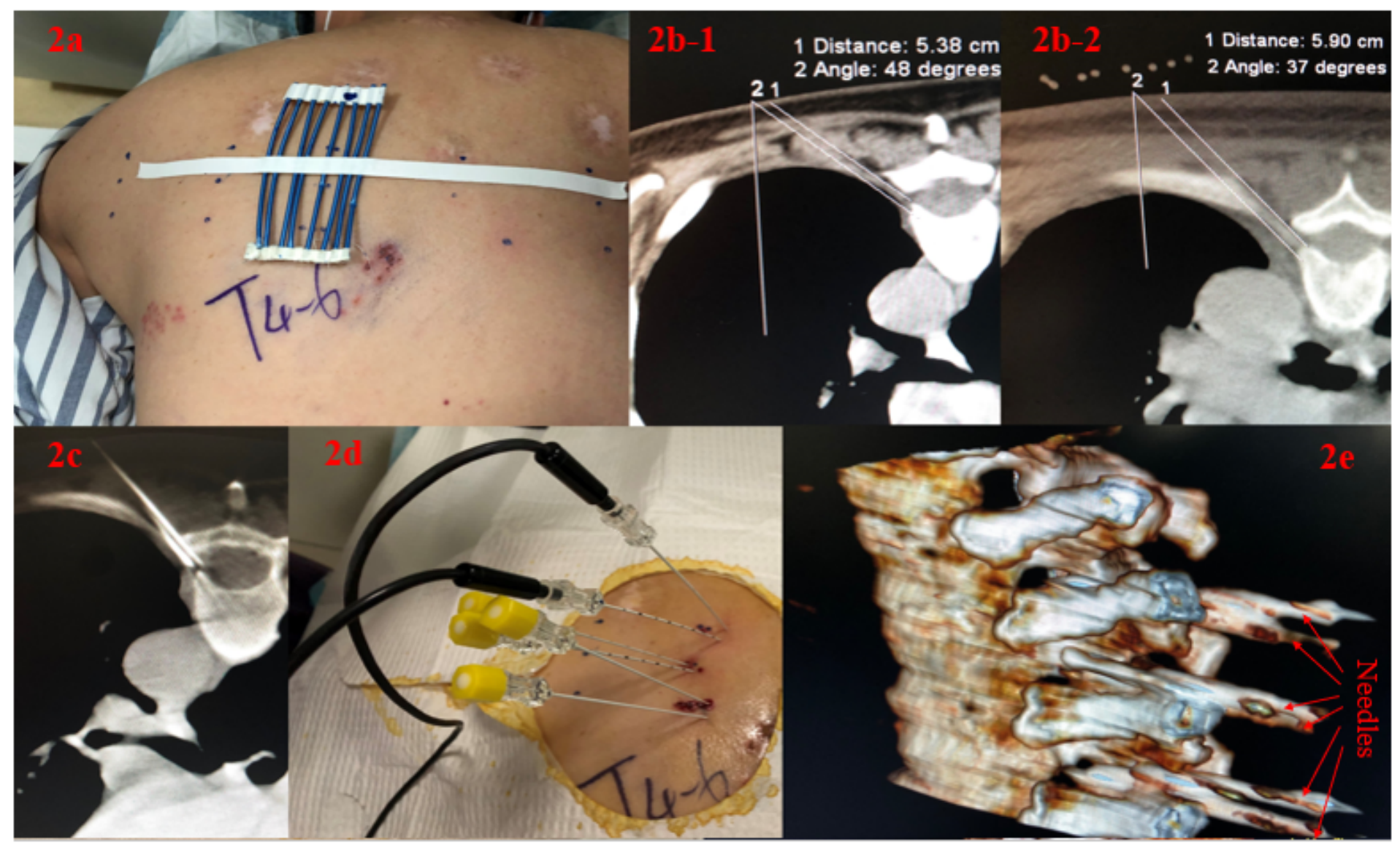

Figure 2

2a: Place positioning mark; $2 \mathrm{~b}-1$ and $2 \mathrm{~b}-2$ : Design the puncture routefrom different cross sections; $2 \mathrm{c}$ : Puncture to predetermined position; $2 \mathrm{~d}$ : Adjust the puncture needle; $2 \mathrm{e}: 3 \mathrm{D}$ reconstruction image of the DCRF group 

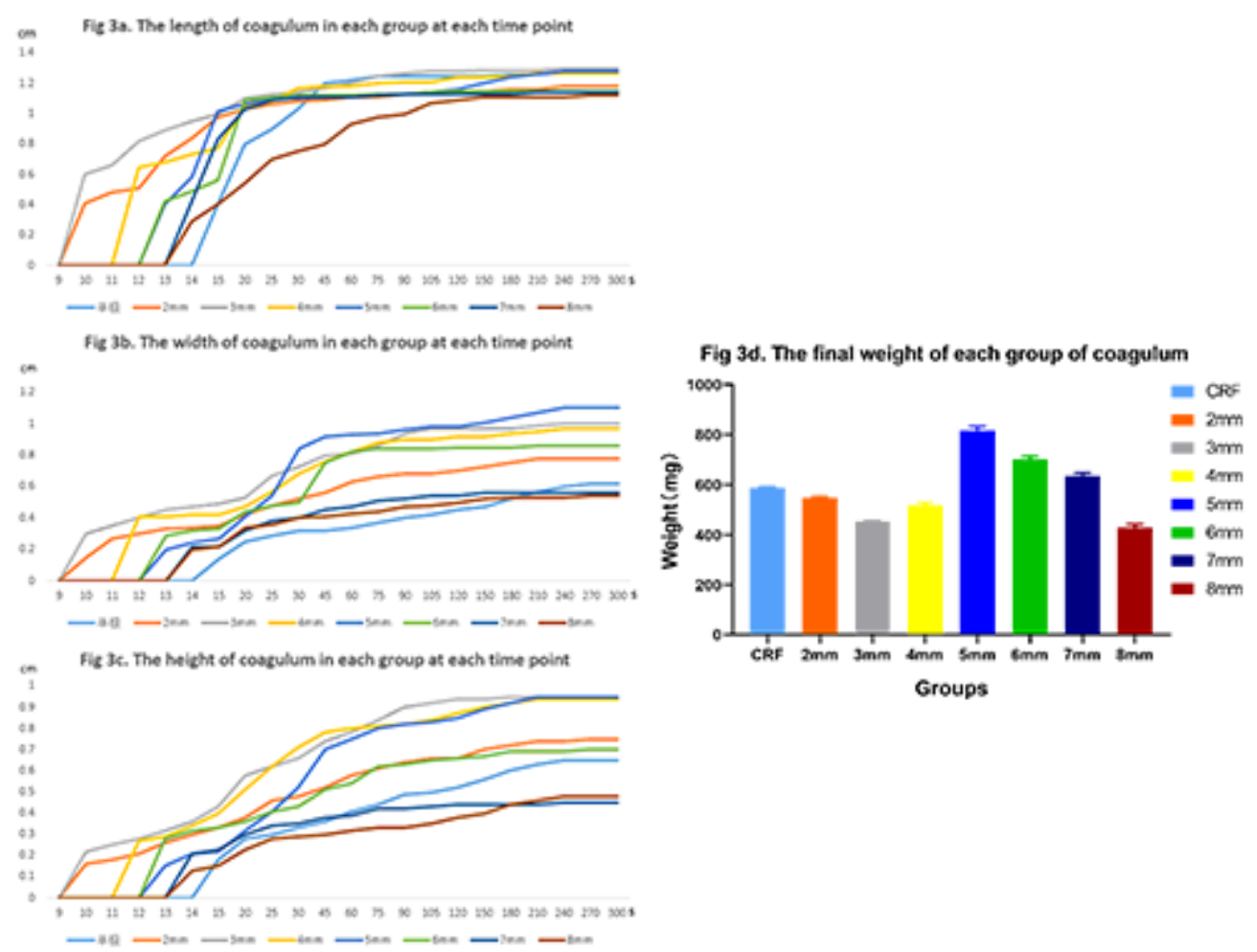

Figure 3

The length, width, height and weight of coagulum in each group

Fig 4. Comparisons of NRS between CRF and DCRF

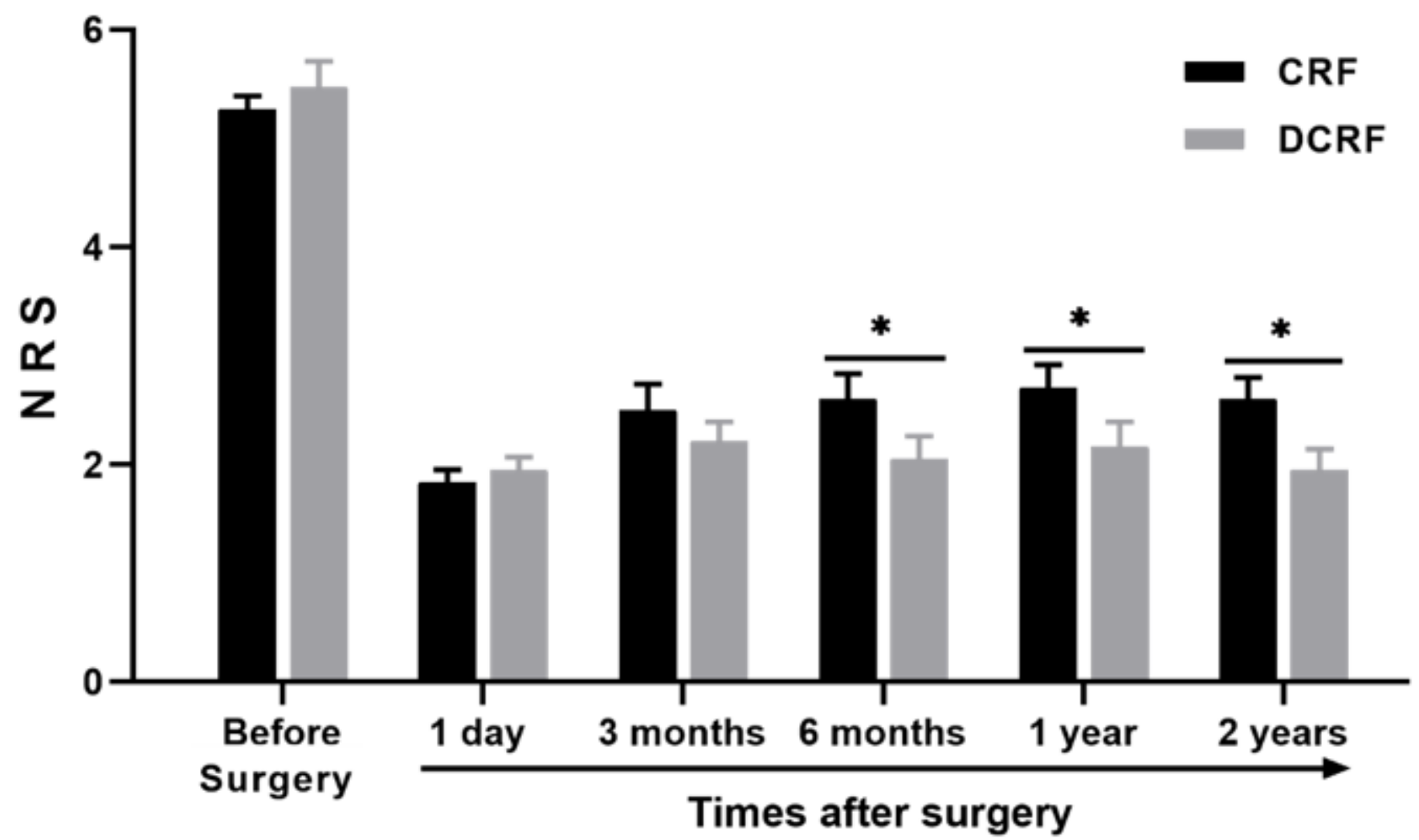




\section{Figure 4}

Comparisons of NRS between CRF and DCRF. At all the time points from 6 months to 2 years following the operation, the NRS score of the DCRF group was significantly lower than that of the CRF group P (6 months $)=0.024 ; \mathrm{P}(1$ year $)=0.041 ; \mathrm{P}(2$ years $)=0.050$ * means $\mathrm{P} \otimes 0.05 ;$ ** means $\mathrm{P} \otimes 0.01$.

\section{Supplementary Files}

This is a list of supplementary files associated with this preprint. Click to download.

- SubsidiaryVideo2DCRF5mm.MOV

- SubsidiaryVideo2DCRF5mm.MOV

- SubsidiaryVideo1DCRF5mm.mp4

- SubsidiaryVideo1DCRF5mm.mp4

- SubsidiaryFigures.docx

- SubsidiaryFigures.docx

- SubsidiaryTables.docx

- SubsidiaryTables.docx 